\title{
Saving Babies? The Consequences of Newborn Genetic Screening
}

\author{
by Stefan Timmermans and Mara Buchbinder \\ Chicago: University of Chicago Press 2012 \\ ISBN 978-0-22692-497-7 \\ Hardcover, US\$30, 320 pp.
}

\author{
Reviewed by B.M. Knoppers \\ Canada Research Chair in Law and Medicine \\ Director, Centre for Genomics and Policy, McGill University, Montreal
}

Starting this review with a synopsis of their conclusion allows us to better situate our overview and analysis. In their conclusion, the authors posit "five contemporary omens that signal the possible futures of newborn screening" (NBS; p. 212): 1) NBS succeeds in the basic goal of identifying rare conditions; 2) NBS will continue to cast an even wider net in the future; 3) program retrenchement and cost-cutting measures could stifle its continued growth; 4) continued evidence is needed, as well as including NBS naysayers in the conversation; and finally, 5) the possibility of growing public dissatisfaction with NBS based on proprietary concerns about government control of personal information. These balanced conclusions reflect well on the nuanced contents of the book itself - an ethnographic study of the spectrum of effects of NBS. Strong on the risks and pitfalls of NBS, the authors are at pains to point out the present and future conundrums based on their analysis of 193 recorded genetic clinic visits with 75 families between 2007 and 2013. The descriptions of the experiences of these families with NBS and its clinical and psycho-social effects not only make for interesting reading but also reveal its poignant reality.

Congruent with the character and language of ethnographic studies (which are not in themselves exaggerated), the chapter titles or headings, such as "Patients in Waiting," "Collateral Damage," "Normalization as a Moral Project," and "Abduction," reveal, however, the subjective undertones of this book. These undertones are best encapsulated by the authors' remark: "Fishing expeditions for rare diseases may not be the most efficacious way to promote population health" (p. 21). Yet, population newborn screening programmes are not fishing expeditions in the wild; rather, they seek to find the asymptomatic at-risk newborn based on the traditional criteria of sensitivity, specificity, and incidence of treatable conditions in the newborn (Wilson and Junger 1968).

Furthermore, it must be remembered how "unique the democratization of newborn screening is in the context of the US healthcare system" (p. 197) when compared to Canada, Scandinavia, or Europe. Perhaps that is why it attracts so much more criticism in the US - as do vaccination or fluoridation, for example. What could be seen as exceptional in the US would be considered essential and part of an integrated public health system, with clinical care and follow-up for affected newborns, in other countries. The authors fear of public health genomics (PHG) is palpable. This reviewer, however, would see the emergence of PHG as a bulwark against privatization, with its inevitable economic inequities for medical care.

While the authors are attuned to the human and clinical complexities and distressful emotions following confirmatory testing in an affected child (p. 227) that clinicians helping affected families face, they compare this to the blunt pro-screening statements of NBS policy advocates. This polarization of views in the US may well be due to the need in the US to constantly fight for any form of basic universal health programs, hence the stridency of public health advocates. Moreover, they seem surprised that "all the families in [their] study 
regarded the screening program positively" (p. 219). The authors consider the parents to be "moral pioneers" and acknowledge that "one of our goals in writing this book has been to bring a wider range of clinician and family experiences into the open" (p. 238).

Returning to the conclusion, the authors describe among the risks to the future of NBS: "Or, we may enter the world of personalized medicine, with whole genome sequencing at birth" (p. 239). While I do not share their latent cynicism of the bona fide public health rationale of NBS, nor their bewilderment at the fortitude and perseverance of parents with affected children who, irrespective of their difficulties, understand the need for NBS across a whole population, I do share this latter concern surrounding the possible introduction of whole genome sequencing into NBS programs. With decreasing costs and consumer (parental?) demand "to know," the introduction of WGS (whole genome sequencing) may be the final blow to the traditional presumed public health consent model dictated by the immediate health needs of screened, affected newborns. Hopefully, that rationale will endure to underpin a basic universal NBS program for all potentially at-risk newborns. But there is no doubt that parental consent should be required for the (largely undecipherable) pediatric newborn report card that WGS will one day provide.

This book is recommended reading, if only for its behind the scenes "humanization" of the experience of clinicians, parents, and children caught up in testing "positive" after an initial NBS screen. Such human nuances constitute valuable lessons learned as we move into the uncharted territory of NBS with the arrival of WGS. The net value of NBS for children, both as a population and as affected individuals, should not be lost nor held hostage, however, in the miasma of WGS discussions. At a minimum, NBS programmes ensure that every child is seen once and accounted for by a health professional. This is important for the recognition of children as future citizens, and as individuals with their own specific health interests (Convention of the Rights of the Child 1989), as distinct from their parents and their choices or lifestyle. The State as a parens patriae owes children this minimum of health care and protection.

\section{Reference}

Wilson, J.M.G., and G. Jungner. 1968. Principles and Practice of Screening for Disease. Geneva: World Health Organization. 